\title{
Chemistry of Collapse and Disk Accretion
}

\author{
S. B. Charnley \& S. D. Rodgers \\ Space Science Division, NASA Ames Research Center, Moffett Field, \\ CA 94035, USA
}

\begin{abstract}
.
Recent results relevant for the survival and chemical alteration of interstellar material at the nebular accretion shock are summarised.
\end{abstract}

\section{Introduction}

A key issue for the ISM-comet connection is to determine under what conditions volatile interstellar matter could survive the accretion shock and arrive, largely unmodified, in the protosolar nebula (see Ehrenfreund \& Charnley 2000). That is, in which regions of the nebula are infalling gaseous molecules and solid ice mantles processed, survive the shock, and simply recondense as ices of similar composition in the post-shock flow? It is also of great interest to quantify the processing in that material which does experience chemical changes. However, there have been no detailed studies of how the accretion process affects the volatile interstellar molecules, apart from those seeking to establish conditions for vaporization (e.g. Neufeld \& Hollenbach 1994). We have therefore begun an extensive study aimed at understanding precisely how interstellar molecules, and their isotopic fractionation ratios, are modified as they collapse towards a protostellar disk, pass through the accretion shock, and subsequently take part in nebular chemistry (see also Markwick \& Charnley in this volume).

Detailed chemical collapse calculations show that heating of the infalling gas by radiation from the protostar/disk leads to evaporation of molecular ices, and thence to strong spatial and temporal molecular abundance gradients in the protostellar core (Rodgers \& Charnley 2003). These calculations indicate that, soon after an 'inside-out' collapse (Shu 1977) has started, infall time-scales become shorter than most chemical time-scales. This results in material from the cool envelope collapsing onto the central protostar without significant chemical alteration. We have extended this into a two-stage model. The first stage is a rotating collapse to allow the composition of accreted interstellar material to be studied as a function of entry position on the nebular disk (Cassen \& Moosman 1981). In this short contribution we present some preliminary results from our modeling of the second stage: chemistry at the accretion shock.

\section{Accretion Shock Chemistry}

We initially consider a simple scenario. As cold interstellar gas and ice-mantled dust grains collapse onto the luminous protostar/disk system they are heated and 
the ice molecules evaporate (e.g. Chick \& Cassen 1997). We take the pre-shock chemical composition to be that corresponding to the general composition of interstellar ice mantles. At the accretion shock, the gas is heated and compressed and we follow the post-shock chemistry of volatile material as it cools and recondenses onto dust grains. For the shock calculations we use a steady interstellar J-shock chemical code (Charnley et al. 1988), based on the model of Hollenbach \& McKee (1979) suitably modified to treat the more extreme conditions of the accretion shock problem (e.g. 3-body reactions, gas-grain interaction, collisional dissociation of $\mathrm{H}_{2}$ ). The chemical network is that of Charnley (1997) but with additional high-temperature reactions from the literature (e.g. for methanol).

\section{Results \& Discussion}

For a given mass accretion rate, the accretion shock is fully dissociative closer to the protostar. Slow shock speeds and low pre-shock densities favor the survival of the most volatile material; these conditions occur in the outer disk. At these and intermediate disk radii, the shock is only partially-dissociative and so a key parameter for the chemistry is the $\mathrm{H} / \mathrm{H}_{2}$ ratio in the post-shock gas. This can increase by many orders of magnitude from its value in quiescent dense gas.

Figure 1 shows the post-shock chemistry for three shock speeds impacting on gas in the outer disk. As expected, the slowest shock produces little chemical change. This preliminary calculation shows that $\mathrm{CO}$ and $\mathrm{H}_{2} \mathrm{O}$ can survive the accretion shock and in fact increase in abundance. Any chemical erosion of $\mathrm{H}_{2} \mathrm{O}$ to $\mathrm{OH}$ and $\mathrm{O}$ by $\mathrm{H}$ atom abstraction reactions is overwhelmed by hydrogenation reactions with $\mathrm{H}_{2}$. The abundances of some molecules, like $\mathrm{CH}_{4}$ and $\mathrm{CO}_{2}$, are eroded in such a J-shock but can partially recover. Other molecules, such as $\mathrm{H}_{2} \mathrm{~S}$, $\mathrm{CH}_{3} \mathrm{OH}$, OCS and $\mathrm{H}_{2} \mathrm{CO}$ are completely destroyed. The post-shock chemistry is highly nonlinear. At the intermediate shock speed the methane abundance actually rises because $\mathrm{CH}_{3} \mathrm{OH}$ is being broken into $\mathrm{CH}_{3}$ and $\mathrm{OH}$; at these postshock temperatures the reaction of $\mathrm{CH}_{3}$ with $\mathrm{H}_{2}$ is faster than its destruction by $\mathrm{H}$ atoms.

In future work we plan to perform a comparative study of the chemistry in dense C-shocks (e.g. Charnley \& Kaufman 2000) since the physical conditions in these shocks may be more benign to molecule survival. We will also make detailed models of specific shock chemistries. This project will eventually allow us, for example, to assess how interstellar deuterium fractionation signatures were altered upon incorporation into the protosolar nebula.

This work was supported by NASA's Origins of Solar Systems and Long Term Space Astrophysics Program's with funds allocated by NASA Ames under Interchange No. NCC2-1412.

\section{References}

Cassen, P. \& A. Moosman 1981, Icarus 48, 353

Chick, K. M. \& Cassen, P. 1997, ApJ, 477, 398

Charnley, S. B. 1997, ApJ, 481, 396

Charnley, S. B., Dyson, J. E., Hartquist, T. W. \& Williams, D. A. 1988, MNRAS231, 269 

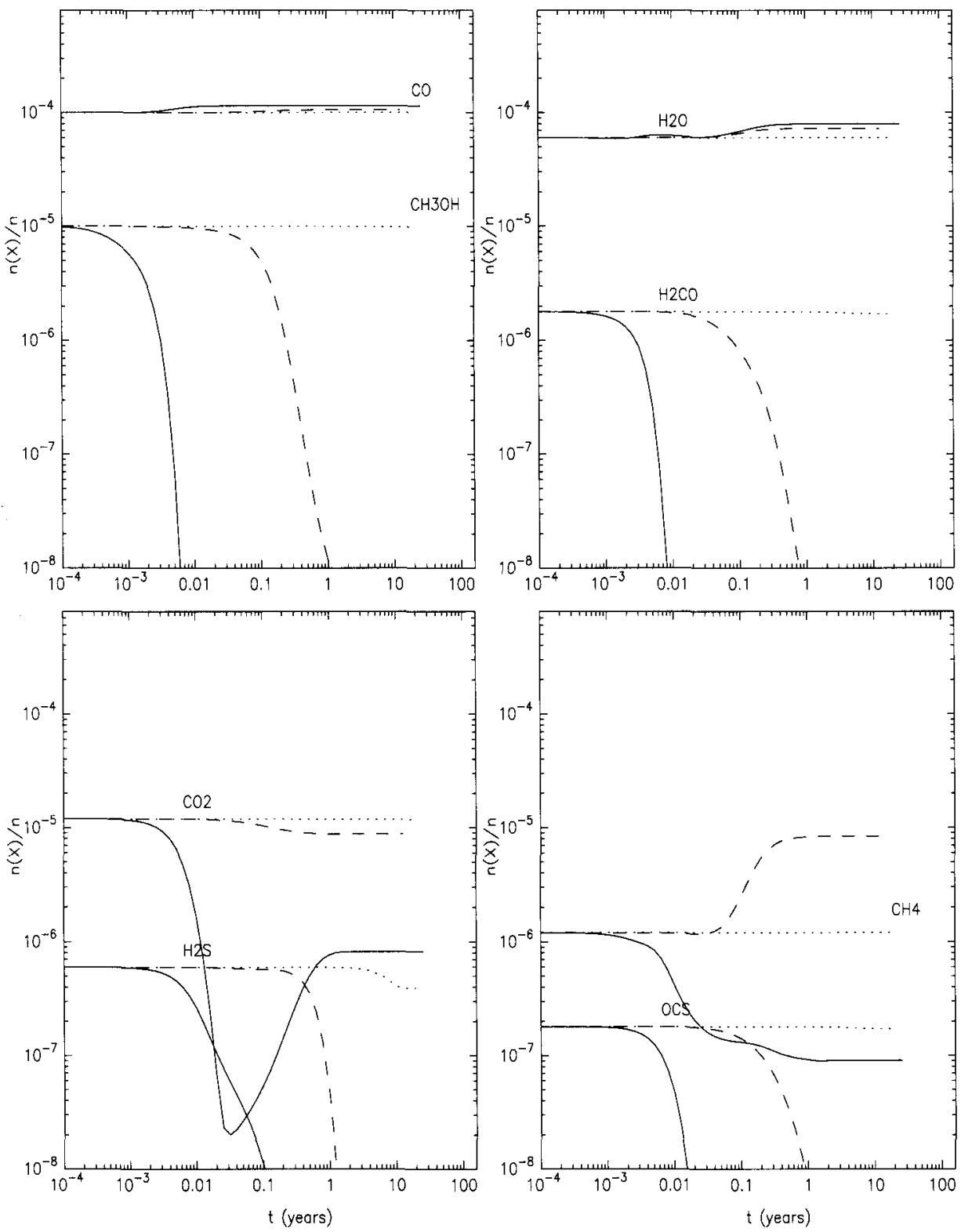

Figure 1. Post-shock chemical evolution of interstellar volatiles at the outer disk accretion shock for a pre-shock gas density of $2 \times 10^{6} \mathrm{~cm}^{-3}$ and shock speeds of $5 \mathrm{kms}^{-1}$ (dotted curves), $8 \mathrm{kms}^{-1}$ (broken curves), and $10 \mathrm{kms}^{-1}$ (solid curves) 
Charnley, S. B., \& Kaufman, M. J. 2000, ApJ, 529, L111

Ehrenfreund, P., \& Charnley, S. B., 2000, ARA\&A, 38, 427

Hollenbach, D. \& McKee, C. F. 1979, ApJS, 41, 555

Neufeld D. \& Hollenbach D. J. 1994, ApJ, 428, 170

Rodgers, S. D., \& Charnley, S. B. 2003, ApJ, 585, 355

Shu, F. H. 1977, ApJ, 214, 488 